\title{
Síntesis de cinamato de metilo, a través de un procedimiento de bajo impacto ambiental, y evaluación de su actividad antifúngica como potencial preservante de maderas
}

\section{Green preparation of methyl cinnamate and evaluation of its antifungal activity as a potential wood preservative}

\author{
Elizabeth Hauga \\ Diego Ruiz ${ }^{\mathrm{b}}$ \\ Natalia Raffaeli ${ }^{c}$ \\ Mario Saparrat ${ }^{\mathrm{d}, *}$ \\ Gustavo Romanelli
}

Fecha de Recepción: 16.11.19

Fecha de Aceptación: 05.03.19

DOI: https://doi.org/10.19053/01217488.v10.n2.2019.8035

\begin{abstract}
Resumen
La prevención y el control de hongos que atacan madera utilizando agentes sintetizados en el marco de la Química Verde es una estrategia prometedora en el desarrollo de preservantes que conduzcan a la sostenibilidad ambiental. La madera constituye un material susceptible a la degradación fúngica, presentando deterioro frecuente y agresivo cuando es puesta en servicio. Si bien a lo largo de los años se han desarrollado compuestos para su protección con diversos métodos de impregnación, muchos de los utilizados presentan fuertes cuestionamientos ambientales. El objetivo de este trabajo fue obtener cinamato de metilo (CiM) a través de un procedimiento de bajo impacto ambiental, y evaluar su actividad in vitro frente a Gloeophyllum sepiarium (Wulf.: Fr.) P. Karst, un hongo causante de pudrición parda. Se realizó la síntesis de (CiM) a partir de ácido cinámico y metanol, mediante catálisis con ácidos de estructura Preyssler, con un rendimiento del 49\% y una selectividad del $100 \%$. Este compuesto ejerció un efecto antifúngico, retrasando el crecimiento miceliar y su velocidad, a partir de una concentración de 2.000 ppm, aunque ello fue dependiente de la temperatura de incubación. Futuros estudios son necesarios para confirmar su potencial antifúngico cuando es aplicado en madera.
\end{abstract}

Palabras clave: antifúngico, cinamato de metilo, preservante, química verde.

a Ingeniera Forestal, Curso de Industrias de Transformación Química. Facultad de Ciencias Agrarias y Forestales. Universidad Nacional de La Plata, Argentina.

b Doctor de la Facultad de Ciencias Exactas, Centro de Investigación en Sanidad Vegetal (CISaV) Facultad de Ciencias Agrarias y Forestales. Universidad Nacional de La Plata, Argentina.

c $\mathrm{PhD}$ in Forest Resources. Laboratorio de Investigaciones en Madera (LIMAD). Universidad Nacional de La Plata., Argentina. / Curso de Industrias de Transformación Química. Facultad de Ciencias Agrarias y Forestales. Universidad Nacional de La Plata. Diagonal 113 y 61. La Plata, Argentina.

d Doctor en Ciencias Naturales. Instituto de Fisiología Vegetal (INFIVE), Universidad Nacional de La Plata (UNLP)-CCT-La Plata-Consejo Nacional de Investigaciones Científicas y técnicas (CONICET), , Argentina / Instituto de Botánica Spegazzini, Facultad de Ciencias Naturales y Museo, Universidad Nacional de La Plata / Cátedra de Microbiología Agrícola, Facultad de Ciencias Agrarias y Forestales, Universidad Nacional de La Plata.

*masaparrat@fcnym.unlp.edu.ar

e Doctor en Química, Centro de Investigación en Sanidad Vegetal (CISaV) Facultad de Ciencias Agrarias y Forestales. Universidad Nacional de La Plata, Argentina / Centro de Investigación en Ciencias Aplicadas "Jorge Ronco", CICCONICET-UNLP. 


\begin{abstract}
Wood constitutes a material susceptible to fungal degradation, presenting frequent and aggressive deterioration when it is put into service. Although over the years compounds have been developed for their protection with various methods of impregnation, many of them present strong environmental questions. The present work focuses on obtaining a preservative with potential antifungal activity for wood through a procedure of low environmental impact. The synthesis of methyl cinnamate (CiM) from cinnamic acid and methanol was carried out by catalysis with acids of Preyssler structure, with a yield of $49 \%$ and a selectivity of $100 \%$. Subsequently, the antifungal action of the compound against Gloeophyllum sepiarium (Wulf.: Fr.) P. Karst, fungus causing brown rot was evaluated in-vitro. To do this, the growth of the fungus was comparatively analyzed on a control agarized culture medium and in the presence of CiM. The CiM demonstrated antifungal effect from $2000 \mathrm{ppm}$, though it was dependent on the incubation temperature. Further research is needed on wood to confirm its potential as a green preservative.
\end{abstract}

Keywords: antifungal, methyl cinamate, preservative, green chemistry.

\title{
1. INTRODUCCIÓN
}

La madera constituye un material con amplia variedad de usos y con claras ventajas frente a otros materiales. Puede ser utilizada como material estructural, combustible o materia prima en industrias, en su forma básica inalterada (construcción, mueblería, postes, leña, etc.), o como subproducto (tableros, pulpa, papel, briquetas, etc.). Se encuentra disponible en diversas formas y tamaños; es posible trabajarla fácilmente con diversas herramientas; presenta gran resistencia en relación al peso, baja conductividad térmica, acústica y eléctrica, alta resistencia a la flexión estática y dinámica y una relativa durabilidad frente a la acción de sustancias químicas o agentes de deterioro atmosférico como los rayos UV, lluvia, etc., presentando mayor resistencia a la oxidación y corrosión. Sin embargo, presenta susceptibilidad frente a la acción de agentes bióticos (hongos, bacterias, insectos y taladradores marinos), viéndose afectadas sus propiedades físicas, químicas, mecánicas y estéticas, y abióticos, sufriendo desgaste químico o mecánico [1]. Si bien las maderas naturalmente tienen cierta durabilidad, dada fundamentalmente por su composición química, generalmente resulta necesaria la adopción de medidas conocidas como métodos de preservación, que permitan protegerlas de la degradación biótica y abiótica, incrementando su durabilidad y resistencia, por ende, su vida útil una vez puesta en servicio $[1,2]$. La degradación por hongos xilófagos representa las mayores pérdidas en durabilidad y resistencia, ya que son atacados los componentes principales de la pared celular (celulosa, hemicelulosas y lignina). Según el grado y modo de degradación de los mismos se identifican tres tipos de pudrición: parda o cúbica, blanca o fibrosa y blanda, siendo la primera la más agresiva, ya que reduce considerablemente la vida útil de la madera en servicio. Desde los estadios iniciales del proceso, las propiedades de resistencia mecánica disminuyen severamente, no siendo proporcional a la pérdida de peso [3]. Mediante la aplicación de sustancias preservantes tanto en superficie como en el interior de la madera, es decir, productos químicos que sean tóxicos para los organismos degradadores pero que no generen daños a la madera, es posible reducir la susceptibilidad de la misma frente a este tipo de degradación [2]. Actualmente, existen en el mercado numerosos productos utilizados con este fin. Sin embargo, algunos de ellos han sido objeto de restricciones en cuanto a sus aplicaciones y comercialización debido a problemas de seguridad y toxicidad tanto ambiental como humana, y persistencia en el ambiente. El arseniato de cobre cromatado (CCA) es un compuesto hidrosoluble ampliamente utilizado a escala mundial y nivel industrial por su alta efectividad como antifúngico e insecticida y buena capacidad de fijación, brindando protección a la madera a largo plazo. Aun teniendo buena fijación, con el paso del tiempo y en particular en ambientes ácidos puede ocurrir la lixiviación de sus componentes, entre ellos, el arsénico inorgánico, muy peligroso para la salud. Como ejemplos de compuestos orgánicos óleo-solubles de alta toxicidad y gran poder de penetración se encuentran el 
pentaclorofenol (PCP) y 2-(tiocianometiltio) benzotiazol (TCMTB); en el caso del PCP, su uso y/o comercialización ha sido prohibido o restringido por su elevada peligrosidad para la salud y el ambiente. Si bien muchos países han reemplazado productos por alternativos considerados "menos dañinos", en ocasiones éstos resultan más costosos y se posee escaso conocimiento respecto a su comportamiento e impacto ambiental. Como ejemplo, se pueden mencionar el compuesto cúprico de amonio cuaternario (ACQ) y el óxido de cromo, cobre y boro (CCB) como reemplazos alternativos del CCA. A raíz de esto, en los últimos años ha ido creciendo el interés por el desarrollo de nuevos compuestos no tóxicos y biodegradables que puedan ser utilizado en la preservación de la madera [2, 4]. En la necesidad de buscar otras alternativas, hemos encontrado que algunos aceites esenciales extraídos de ciertas especies vegetales (particularmente del género Ocimum), utilizados como repelentes, insecticidas, antimicrobianos $\mathrm{y}$ antioxidantes, $\mathrm{y}$ que han evidenciado un importante efecto fungitóxico sobre ciertos hongos que afectan a cultivos agrícolas, contienen en su composición una elevada concentración de un derivado del ácido cinámico: el cinamato de metilo (CiM) [5]. Esto último también se verifica en aceites esenciales extraídos de especies del género Cinnamomum [6], con elevada actividad antifúngica sobre diversos hongos. Además, existen antecedentes de este compuesto como potente nematicida [7] y como repelente de colémbolos micófagos (Hexapoda:
Entognatha) - microartrópodos hexápodos - a elevadas concentraciones [8]. Si bien aún no se han hallado pruebas de su utilización como agente antifúngico en madera, podría constituir un potencial principio activo en formulaciones preservantes de bajo impacto ambiental teniendo en cuenta, además, su baja persistencia en el ambiente, baja toxicidad para organismos no blanco, y mayor especificidad en su modo de acción, por tratarse de un compuesto de origen botánico [9]. El CiM puede obtenerse a partir de ciertas especies vegetales que lo sintetizan naturalmente. Sin embargo, para su empleo a gran escala en dichas formulaciones se debe recurrir a la síntesis en laboratorio, a fin de obtener un mayor rendimiento. Para ello, en relación a la búsqueda de alternativas más amigables humana y ambientalmente, el uso de la Química Verde presenta claras ventajas respecto a la química tradicional. Mediante el diseño de productos y procesos químicos, aplicando doce principios, es posible reducir o eliminar la producción y el uso de sustancias peligrosas para el medio ambiente y la salud humana, teniendo en cuenta desde el inicio del proceso, el impacto que puede implicar una nueva sustancia en ambas dimensiones [10].

El objetivo general del trabajo fue sintetizar en laboratorio cinamato de metilo (CiM) en el marco de la Química Verde y evaluar su actividad in vitro frente a Gloeophyllum sepiarium (Wulf.: Fr.) P. Karst, un hongo causante de pudrición parda, a dos temperaturas de incubación.

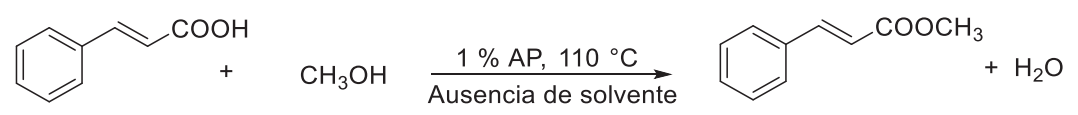

Figura 1. Síntesis de cinamato de metilo.

\section{METODOLOGÍA}

\subsection{General}

Los productos químicos utilizados fueron de grado analítico y empleados sin posterior purificación. El producto fue identificado por la determinación del punto de fusión del compuesto el cual fue determinado por el método del capilar y la caracterización espectroscópica se llevó a cabo por la determinación de los espectros de ${ }^{1} \mathrm{H}-\mathrm{RMN}$ y ${ }^{13} \mathrm{C}-\mathrm{RMN}$. Los resultados fueron similares a los reportados en la literatura [11] $]^{1}$.

1 El CiM, ${ }^{1} \mathrm{H}$ NMR (400 MHz, $\left.\mathrm{CDCl}_{3}\right): \delta 3.80(3 \mathrm{H}, \mathrm{s}$, $\left.\mathrm{OCH}_{3}\right), 6.44\left(1 \mathrm{H}, \mathrm{d}, \mathrm{J}=16.0 \mathrm{~Hz}, \mathrm{MeOCCH}_{-}\right), 7.37-7.39$ $(3 \mathrm{H}, \mathrm{m}, \mathrm{Ar}-\mathrm{H}), 7.51-7.52(2 \mathrm{H}, \mathrm{m}, \mathrm{Ar}-\mathrm{H}), 7.70(1 \mathrm{H}, \mathrm{d}, \mathrm{J}=$ $\left.16.0 \mathrm{~Hz}, \mathrm{ArCH}_{=}\right)$.

El CiM obtenido con cinamaldehído y metanol [10] arrojó ${ }^{1} \mathrm{H}$ NMR (400 MHz, $\left.\mathrm{CDCl}_{3}\right) \delta 7.70(1 \mathrm{H} \mathrm{d}, \mathrm{J}=16.0$ 
Síntesis del catalizador (Ácido de Preyssler, $\left.\boldsymbol{H}_{14}\left[\mathrm{NaP}{ }_{5} \boldsymbol{W}_{29} \mathrm{MoO}_{110}\right]\right):$ se siguió esencialmente un procedimiento descrito en la literatura [12].

Síntesis de cinamato de metilo se colocaron en un recipiente de reacción (Tubo de acero inoxidable sellado) 1 milimol de ácido transcinámico y $4 \mathrm{ml}$ de metanol, junto a $1 \mathrm{mmol} \%$ de $\mathrm{AP}$, en un baño de vaselina calentado a $110^{\circ} \mathrm{C}$, con agitación. El avance de la reacción se observó realizando una cromatografía en capa delgada cada una hora, usando tolueno como solvente de desarrollo. No se observaron cambios en la composición de la mezcla luego de 16 horas de reacción. El cinamato de metilo se extrajo con 2 $\mathrm{ml}$ de tolueno y se lavó con porciones de solución de $\mathrm{NaHCO}_{3}$ al $10 \%$ ( $3 \times 2 \mathrm{ml}$ ), y luego con agua destilada $(2 \times 2 \mathrm{ml})$, y la fase orgánica se secó con $\mathrm{Na}_{2} \mathrm{SO}_{4}$ anhidro. La solución en tolueno con el cinamato de metilo se diluyó con $3 \mathrm{ml}$ de éter de petróleo, y se resolvió mediante cromatografía en columna utilizando como solvente una mezcla 1:1 de éter de petróleo y tolueno. El solvente de las fracciones reunidas se evaporó en vacío, hasta que el residuo de la evaporación alcanzó peso constante. El compuesto fue obtenido con un rendimiento del $49 \%$ (selectividad $100 \%$ ) libre de productos secundarios.

\subsection{Bioensayos}

Material fúngico $\mathrm{Se}$ utilizó el aislamiento Gloeophyllum sepiarium (Wulf.: Fr.) P. Karst. LPSC 735 (Cepario del Instituto Spegazzini, Facultad de Ciencias Naturales y Museo, UNLP, La Plata), un hongo causante de pudrición parda sobre madera que frecuentemente está asociada al deterioro de la madera en servicio [3]. Los cultivos stock del hongo sobre el medio agar-extracto de malta (2 \%; medio basal, MB) se mantuvieron en estrías a $4^{\circ} \mathrm{C}$ siguiendo a Saparrat et al. [13].

Evaluación de la actividad antifúngica del CiM en cultivo agarizado El ensayo se realizó en placas de Petri conteniendo MB y en presencia de un rango de concentración de CiM, entre 500 y 4000 ppm, así como de un control negativo con el solvente (acetona) y de un control positivo

$\mathrm{Hz}), 7.54-7.49$ (2 H, m), 7.41-7.35 (3 H, m), 6.44 (1 H, d, $\mathrm{J}=16.0 \mathrm{~Hz}), 3.80(3 \mathrm{H}, \mathrm{s}) .{ }^{13} \mathrm{C} \mathrm{NMR}\left(101 \mathrm{MHz}, \mathrm{CDCl}_{3}\right)$ $\delta 167.5,144.9,134.5,130.4,129.0,128.1,117.9,51.8$. con IPBC (3-yodo-2-propinil butil carbamato, $\mathrm{C}_{8} \mathrm{H}_{12} \mathrm{INO}_{2}$ ) a $100 \mathrm{ppm}$ como un fungicida de referencia. Las placas fueron inoculadas con un disco de micelio del hongo de $0,6 \mathrm{~cm}$ de diámetro (obtenido a partir de cultivos agarizados sobre medio basal después de 7 días de incubación), e incubadas a $22 \pm 2^{\circ} \mathrm{C}$ y $27 \pm 2^{\circ} \mathrm{C}$ durante 26 días bajo oscuridad. Periódicamente (a intervalos de 2-3 días) se evaluó el tiempo necesario desde la inoculación para la colonización del hongo sobre cada medio obtenido (fase de latencia $\lambda$ ) y el diámetro de la colonia resultante, siendo estimada la concentración Mínima Inhibitoria del CiM (CMI) y la tasa de crecimiento $\mathrm{K}$ ( $\mathrm{cm} /$ día) siguiendo a Troncozo et al. [14]. Se realizó un análisis de la varianza de los datos correspondiente a cada tratamiento $(p<0,05)$ utilizando InfoStat [15].

\section{RESULTADOS Y DISCUSIÓN}

\subsection{Síntesis del cinamato de metilo}

Se llevó a cabo mediante una metodología que incluye la utilización de un catalizador heteropoliácido de estructura tipo Preyssler $\left(\mathrm{H}_{14} \mathrm{NaP}_{5} \mathrm{MoW}_{29} \mathrm{O}_{110}\right)$. La reacción consiste en una esterificación de Fischer entre el ácido cinámico y el metanol y se lleva adelante en ausencia de solvente. (Esquema 1). Diferentes condiciones de reacción fueron chequeadas, las cuales incluyen el efecto de la temperatura, la cantidad de catalizador y el tiempo de reacción. Las condiciones óptimas de síntesis de cinamato de metilo se obtienen a $110{ }^{\circ} \mathrm{C}$, en ausencia de solvente, empleando un $1 \%$ en milimol de AP, en un período de tiempo de 24 horas, empleando como reactor un tubo de acero inoxidable cellado. En tales condiciones el cinamato de metilo se obtiene con un rendimiento del $49 \%$ (conversión $49 \%$, selectividad 100\%), libre de productos secundarios. El catalizador utilizado puede recuperarse y reciclarse sin apreciable pérdida de la actividad catalítica.

\subsection{Evaluación de la actividad antifúngica del CiM en cultivo agarizado}

El CiM ejerció un efecto fungistático sobre $G$. sepiarium, limitando el crecimiento del micelio fúngico a medida que aumentó su concentración en el medio (Figuras 2 y 3 ) (Tabla 1), existiendo diferencias estadísticamente significativas entre los tratamientos $(\mathrm{p}<0,001)$. 


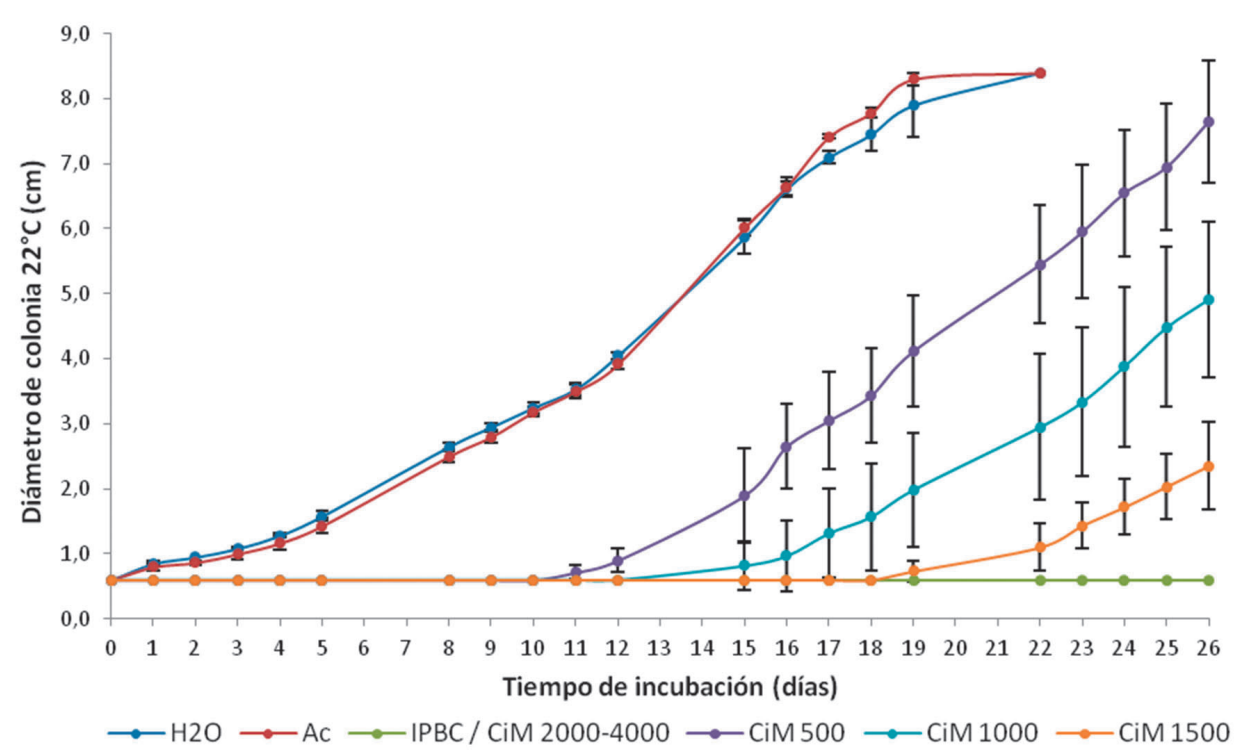

Figura 2. Cinética de crecimiento miceliar de G. sepiarium en medios de control (agua y acetona) y en presencia de CiM a 500-4000 ppm, e IPBC a $100 \mathrm{ppm}$ a $22^{\circ} \mathrm{C}$. Los datos registrados y barras de error (Desviación Estándar) corresponden

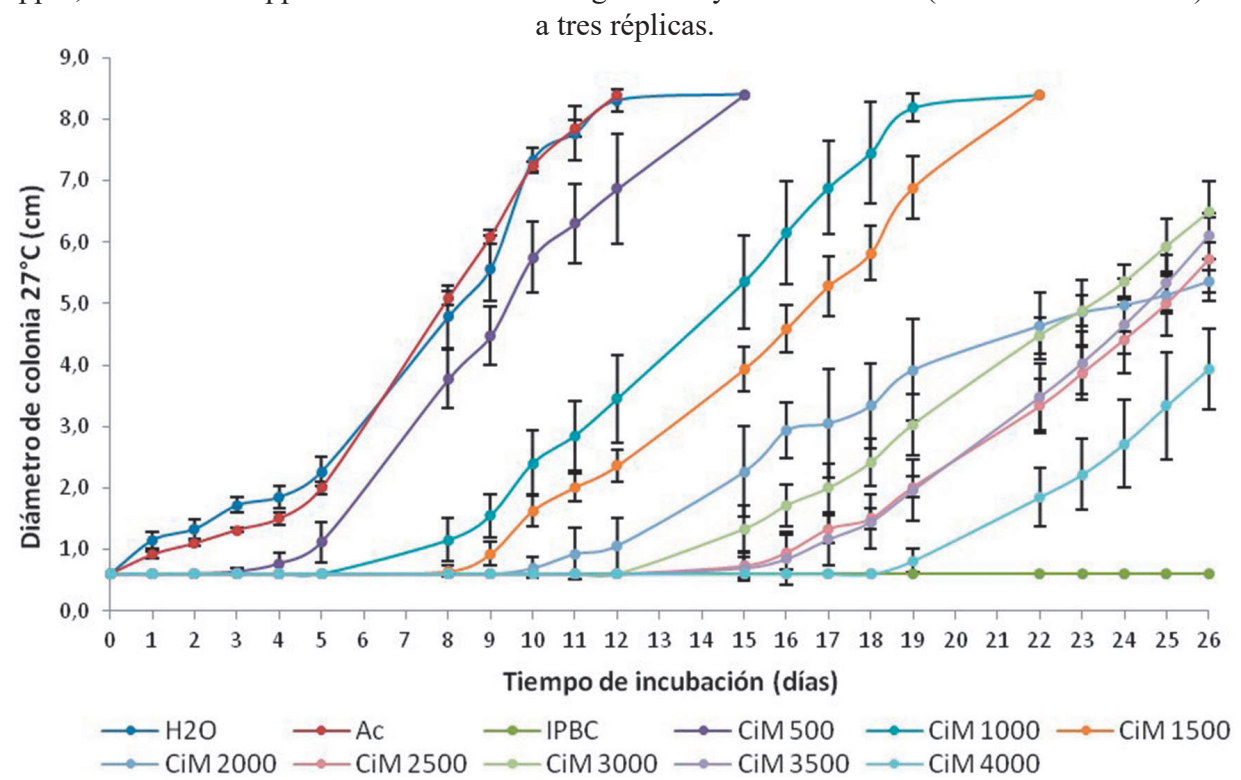

Figura 3 - Cinética de crecimiento miceliar de G. sepiarium en medios de control (agua y acetona) y en presencia de CiM a 500-4000 ppm, e IPBC a 100 ppm a $27^{\circ} \mathrm{C}$. Los datos registrados y barras de error (Desviación Estándar) corresponden a tres réplicas.

Tabla 1 - parámetros estadísticos de las rectas de regresión lineal entre el diámetreo de las colonias de $G$. sepiarium sobre medios de control (agua y acetona) y en presencia de CiM a 500-4000 ppm, e IPBC a 100 ppm (variable dependiente) versus el tiempo de incubación (variable independendiente).

\begin{tabular}{l|c|c|c|c}
\hline \multirow{2}{*}{ Tratamiento } & \multicolumn{2}{|c|}{$\boldsymbol{K}(\mathbf{c m} /$ día) } & \multicolumn{2}{c}{$\lambda$ (días) } \\
\cline { 2 - 5 } & $\mathbf{2 2 ^ { \circ } \mathbf { C }}$ & $\mathbf{2 7}^{\circ} \mathbf{C}$ & $\mathbf{2 2}^{\circ} \mathbf{C}$ & $\mathbf{2 7}^{\circ} \mathbf{C}$ \\
\hline H2O (Control - a) & 0,483 & 0,905 & 4,0 & 3,2 \\
Ac $($ Control - b) & 0,525 & 0,998 & 4,6 & 3,5 \\
IPBC (Control +) & - & - & - & - \\
CM 500 & 0,487 & 0,742 & 11,9 & 3.8 \\
CM 1000 & 0,388 & 0,646 & 15,4 & 7,4
\end{tabular}




\begin{tabular}{lcccc} 
CM 1500 & 0,235 & 0,571 & 19,1 & 8,6 \\
CM 2000 & - & 0,313 & - & 9,5 \\
CM 2500 & - & 0,451 & - & 15,4 \\
CM 3000 & - & 0,441 & - & 13,2 \\
CM 3500 & - & 0,497 & - & 15,7 \\
CM 4000 & - & 0,441 & - & 18,9 \\
\hline
\end{tabular}

(-): no se registró crecimiento.

A diferencia de los controles negativos, donde se evidenció crecimiento del micelio desde el inicio del ensayo, en los tratamientos con CiM éste se vio retardado y la tasa de crecimiento resultó menor. Se observó que, a medida que aumentó la concentración del compuesto en el medio, la fase de latencia se extendió y la tasa de crecimiento disminuyó. Lo contrario ocurrió teniendo en cuenta la temperatura, donde se registró un acortamiento de la fase de latencia e incremento de la tasa de crecimiento al aumentar la misma. No se registró crecimiento del micelio en los tratamientos de mayor concentración de $\mathrm{CiM}(2000$ a $4000 \mathrm{ppm})$ a $22^{\mathrm{a}} \mathrm{C}$ y en el control positivo. A $22{ }^{\circ} \mathrm{C}$, el CiM demostró efecto antifúngico a partir de 2000 ppm (CMI), observando un retraso del crecimiento a partir de 500 ppm, que sólo fue evidente luego de 12 días respecto a las colonias sobre el medio de cultivo basal. Se comenzó a registrar poder inhibitorio a partir de $1000 \mathrm{ppm}$, con un valor del 44,7\%. A $27{ }^{\circ} \mathrm{C}$, el crecimiento se vio retardado a partir de $1000 \mathrm{pm}$, visualizándose transcurridos los 7 días de iniciado el ensayo. Se registró inhibición a partir de $2000 \mathrm{ppm}$, con un valor del 39,1\%, y el mayor poder inhibitorio se verificó a 4000 ppm, con un valor del 57,3\%. No se observaron diferencias en las características y morfología de la colonia fúngica en los tratamientos con CiM respecto a los controles negativos, aunque sí se evidenció mayor variabilidad en el crecimiento en diámetro entre réplicas de un mismo tratamiento.

En este trabajo se ha estudiado la síntesis de CiM mediante un procedimiento de bajo impacto ambiental y su evaluación como antifúngico, utilizando a $G$. sepiarium como organismo test a controlar, en cultivos agarizados.

El uso de catalizadores heteropoliácidos sólidos como los de Preyssler, constituyen una alternativa prometedora en la síntesis con bajo impacto ambiental, ya que generan una reducida cantidad de residuos y productos secundarios, siendo posible su recuperación y reutilización sin pérdida apreciable de su actividad catalítica. Esto reemplaza el uso de ácidos minerales solubles, cuyo desecho generan residuos contaminantes $[16,17]$.

En el ensayo del hongo sobre medio agarizado, el efecto del CiM sobre el crecimiento de $G$. sepiarium se evaluó analizando la fase de latencia y la tasa de crecimiento de la fase lineal. Teniendo en cuenta que $G$. sepiarium es una especie termotolerante $[18,19]$, con una temperatura óptima de crecimiento entre los $30-50^{\circ} \mathrm{C}$ [20], y debido a que la temperatura es un parámetro físico que condiciona el crecimiento fúngico, se realizaron los ensayos a 22 y $27^{\circ} \mathrm{C}$ de incubación.

Se observó la respuesta diferencial del CiM en su acción inhibitoria sobre el crecimiento de G. sepiarium en función de la temperatura de incubación, con reducción de la fase de latencia, aumento de la tasa de crecimiento y menores porcentajes de inhibición al incrementar la temperatura. Dado que en las condiciones de ensayo el compuesto es estable, el comportamiento diferencial sugiere una activación térmica del metabolismo del hongo a temperaturas próximas al rango de crecimiento óptimo para $G$. sepiarium $\left(30-50^{\circ} \mathrm{C}\right)[20]$. A la vez, una mayor disponibilidad de humedad, producto de la mayor evaporación en el sistema de estudio (cajas de Petri), podría generar mejores condiciones de crecimiento para el desarrollo de micelio [21].

El CiM ejerció un control sobre el crecimiento del hongo, retardando el crecimiento, con menor tasa, y mayor porcentaje de inhibición al aumentar su concentración en el medio. A $22^{\circ} \mathrm{C}$, el CiM demostró efecto antifúngico a partir de 2000 ppm, con retraso del crecimiento (12 días) 
a partir de $500 \mathrm{ppm}$ y poder inhibitorio a partir de 1000 ppm $(44,7 \%)$. A $27^{\circ} \mathrm{C}$, el crecimiento se ve retardado (7 días) a partir de 1000 pm, con inhibición a partir de 2000 ppm $(39,1 \%)$ y mayor poder inhibitorio a $4000 \mathrm{ppm}(57,3 \%)$. Hay reportes sobre la utilización de CiM con distintos fines a menores concentraciones de trabajo. Bin Jantan et al. [6] hallaron una elevada actividad antifúngica sobre hongos no xilófagos por parte del CiM extraído de aceites esenciales de especies del género Cinnamomum, con CMI de $630 \mathrm{ppm}$. Sobre otros organismos, el CiM tiene un efecto tóxico también a concentraciones inferiores, actuando como potente nematicida, con valores de LC507 de 163 ppm [7] y repeliendo colémbolos micófagos a partir de 100 ppm con inhibición incompleta pero significante y fuerte inhibición a partir de 1000 ppm [8]. Comparando estos resultados con los obtenidos en este trabajo y en trabajos previos [22], se observa que la acción antifúngica del CiM sobre G. sepiarium se da a elevadas concentraciones. Esto podría deberse a la existencia de mecanismos detoxificadores de este compuesto por el hongo. Existen reportes sobre la capacidad de algunos hongos de pudrición parda para degradar ciertos compuestos aromáticos. Jawallapersand et al. [23] hacen referencia a la existencia de enzimas con actividad monooxigenasas del complejo P450 de la familia CYP53, con un rol importante en la degradación de compuestos aromáticos y detoxificación de compuestos tóxicos y moléculas intermediarias resultantes, como el benzoato, siendo dicho complejo esencial para la supervivencia de los mismos. Hongos pardos como Postia placenta [24], Tyromyces palustris y Gloeophyllum trabeum [25] (Basidiomycota), cuentan con este sistema, con capacidad de metabolizar compuestos endógenos y exógenos mediante reacciones de hidroxilación.

\section{CONCLUSIONES}

La síntesis del CiM mediante la Química Verde es viable y conlleva numerosas ventajas en comparación con la química tradicional, como la economía atómica, la no utilización de sustancias auxiliares como solventes, la formación de productos biodegradables y de baja toxicidad, entre otros. La reacción llevada a cabo en tubo sellado, en un baño calentado a $110{ }^{\circ} \mathrm{C}$ empleando 1 milimol de ácido trans-cinámico, 4 $\mathrm{ml}$ de metanol, $1 \mathrm{mmol} \%$ de AP, durante 16 horas condujo a la obtención de cinamato de metilo con un rendimiento del $49 \%$ (selectividad 100\%). Según los ensayos realizados el compuesto demuestra actividad antifúngica en un hongo cultivado en medio agarizado a una concentración de 2000 ppm a $22{ }^{\circ} \mathrm{C}$. Si bien el compuesto ha sido ensayado en un hongo causante de pudrición castaña, resultaría interesante analizar el efecto que puede tener sobre hongos xilófagos causantes de los otros dos tipos de pudriciones. Asimismo, en futuras investigaciones resulta necesario probar el compuesto en probetas de madera, bajo distintas condiciones según las especies forestales, variandoel método de aplicación del compuesto, el tamaño de las probetas, etc. Los resultados obtenidos hasta el momento indican un comportamiento promisorio del compuesto sintetizado, y requerirán de investigaciones posteriores, fundamentalmente sobre madera, para poder obtener resultados más fehacientes que determinen que el CiM pueda ser utilizado como un persevante verde.

\section{AGRADECIMIENTOS}

Esta investigación fue parcialmente subsidiada por:

- La Agencia Nacional de Promoción Científica y Tecnológica (ANPCyT) del Ministerio de Ciencia, Tecnología e Innovación Productiva a través del proyecto PICT 2015-1620 (Mario Carlos Nazareno Saparrat), CONICET (PUE INFIVE) y UNLP, Argentina. Mario C. N. Saparrat y Gustavo P. Romanelli son investigadores del CONICET, Argentina.

- Lla Secretaria de Ciencia y Técnica de la Universidad Nacional de La Plata, a través del Proyecto I+D Código 11 A291, período 20162019, Director: Ing. Gabriel D. Keil.

\section{REFERENCIAS}

[1] S. Monteoliva. La madera: propiedades y productos forestales. Apunte de Cátedra de Xilotecnología, Facultad de Ciencias Agrarias y Forestales - UNLP, 2009, 13 pp.

[2] M.C. Murguía, "Desarrollo de nuevos productos para la preservación de maderas", 
en Actas de III Congreso Iberoamericano de Protección de la Madera (CIPROMAD). Concepción, Chile, 2012, 10 pp.

[3] M. Murace, E. Spavento, G.D. Keil, M. Saparrat, "Pudrición castaña: efectos sobre las propiedades de resistencia mecánica de la madera", Revista de Ciencias Forestales - Quebracho, vol. 18 (1,2), 2010, pp.37-46.

[4] G.D. Keil, Biodegradación y Preservación de la Madera: Sustancias Preservantes. Curso de Actualización para Graduados, Universidad Nacional del Comahue. 2004, 9 pp.

[5] E. Murillo, A. Viña, I.L. Correa,"Actividad antifúngica y composición química del aceite esencial de doce variedades de Ocimum sp. cultivadas en Ibague Colombia", Información Tecnológica, vol. $13 \mathrm{~N}^{\circ} 1,2002$.

[6] I. Bin Jantan, B.A.K. Moharam, J. Santhanam, J.A. Jamal, "Correlation Between Chemical Composition and Antifungal Activity of the Essential Oils of Eight Cinnamomum Species", Pharmaceutical Biology, Vol. 46, No. 6, 2008, pp. 406-412.

[7] J-O. Kong, S-M. Lee, Y-S. Moon, S-G. Lee, Y-J. Ahn, "Nematicidal Activity of Cassia and Cinnamon Oil Compounds and Related Compounds toward Bursaphelenchus xylophilus (Nematoda: Parasitaphelenchidae)", Journal of Nematology, vol 39(1), 2007, pp.31-36.

[8] T. Sawahata, S. Shimano, M. Suzuki, "Tricholoma matsutake 1-ocen-3-ol and methyl cinnamate repel mycophagous Proisotoma minuta (Collembola: Insecta)", Mycorrhiza, vol 18, 2008, pp. 111-114.

[9] M.E. Pérez, D. Ruiz, D.;G. Pasquale, F. Barberis, M.I. Schneider, G.P. Romanelli, J.C. Autino, "Preparación de cinamatos de alquilo en condiciones de bajo impacto ambiental",Investigación Joven, vol. 2 (1), 2015, pp. 33.
[10] G. P. Romanelli, Curso de Química Verde: retos para un desarrollo sostenible, Universidad del Cauca, Popayán, Colombia, 2013.

[11] J. Ruan, X. Li, O. Saidi, J. Xiao, "Oxygen and Base-Free Oxidative Heck Reactions of Arylboronic Acids with Olefins", J. Am. Chem. Soc., vol. 130, 2008, 2424-2425.

[12] F.F. Bamoharram, M.M. Heravi, M. Roshani, M. Jahangir, A. Gharib, "Preyssler catalyst, $\left[\mathrm{NaP}_{5} \mathrm{~W}_{30} \mathrm{O}_{110}\right]^{14-}$ : a green, efficient and reusable catalyst for esterification of salicylic acid with aliphatic and benzylic alcohols", Appl. Catal. A Gen., vol. 302, 2006, pp. 42-47.

[13] M.C.N. Saparrat, M.J. Martínez, M.N. Cabello, A.M. Arambarri, "Screening for ligninolytic enzimes in autochthonous fungal strain from Argentine isolated from different substrata", Revista Iberoamericana de Micología, vol. 19, 2002, pp. 181-185.

[14] M.I. Troncozo, R.P. Gómez, A.M. Arambarri, P.A. Balatti, A.M.M. Bucsinszky, M.C.N. Saparrat, "Growth and oxidative enzymatic activity of in-vitro cultures of Ciliochorella buxifolia", Mycoscience, vol. 56, 2015, pp. 58-65.

[15] J.A. Di Rienzo, M. Balzarini, L. González, F. Casanoves, M.M. Tablada, C.W. Robledo, "Software estadístico InfoStat", Universidad Nacional de Córdoba (FCAUNC), 2016.

[16] M.E. Pérez, D. Ruiz, G.P. Romanelli, M. I. Schneider, J.C. Autino, "Bioactividad de derivados naturales del ácido cinámico", XIX Simposio Nacional de Química Orgánica, 2013. Diponible en: http:// www.conicet.gov.ar/new_scp/detalle. php? keywords $=\& \mathrm{id}=05420$ \&inst $=$ yes \& congresos $=$ yes \& detalles $=$ yes $\&$ congr $\mathrm{id}=2195821$. Último acceso: mayo 2018.

[17] D. Ruiz, G. Romanelli, P. Vázquez, J. Autino. J., "Catálisis con heteropoliácidos con estructura de Preyssler. Aplicación en la 
síntesis de fenilpropanoides“, VI Simposio Colombiano de Catálisis. Medellín, Colombia, 2009, 5 pp.

[18]NorthAmerican PlantProtection Organization (NAPPO), "Review of heat treatment of wood and wood packaging“, NAPPO Science and Technology Documents, 2014, pp. 7-8.

[19] R.A. Zabel, J.J. Morrell, "Wood Microbiology: Decay and Its Prevention", Academic Press, Inc. USA, 1992, pp. 106.

[20] G. Alfredsen, H. Solheim, K.M. Jenssen, "Evaluation of decay fungi in Norwegian buildings", International Research Group on Wood Protection. India. IRG/WP 0510562, 2005, 12 pp.

[21] T. Stienen, O. Schmidt, T. Huckfeldt, "Wood decay by indoor basidiomycetes at different moisture and temperature“, Holzforschung, vol. 68(1), 2014, pp. 9-15.

[22] E. Haug, N. Raffaeli, G. Romanelli, D. Ruiz, M. Saparrat, G. Keil, "Síntesis de Bajo Impacto Ambiental de un Preservante de Maderas a partir de la Química Verde“, V
Congreso Iberoamericano de Protección de la Madera. Colonia de Sacramento, Uruguay, 2016, 9 pp.

[23] P. Jawallapersand, S.S. Mashele, L. Kovac`ič, J. Stojan, R. Komel, S.B. Pakala, N. Kraševec, K. Syed, "Cytochrome P450 Monooxygenase CYP53 Family in Fungi: Comparative Structural and Evolutionary Analysis and Its Role as a Common Alternative Anti-Fungal Drug Target", PLoS ONE, vol. 9(9), 2014, e107209, 15 pp.

[24] J. Zhang, G.N. Presley, K.E. Hammel, J-S. Ryu, J.R. Menke, M. Figueroa, D. Hu, G. Orr, J.S. Schilling, "Localizing gene regulation reveals a staggered wood decay mechanism for the brown rot fungus Postia placenta", Proceedings of the National Academy of Sciences, vol. 113(39), 2016, pp. 10968-10973.

[25] F. Kamada, S. Abe, N. Hiratsuka, H. Wariishi, H. Tanaka, "Mineralization of aromatic compounds by brown-rot basidiomycetesmechanisms involved in initial attack on the aromatic ring", Microbiology, vol. 148(6), 2002, pp. 1939-1946. 